\title{
Evaluating the BioSense Syndrome for Heat-Related IIIness in Maricopa County, Arizona
}

\author{
Jessica R. White*, Kate Goodin and Vjollca Berisha \\ Epidemiology, Maricopa County Department of Public Health, Phoenix, AZ, USA
}

\section{Objective}

To evaluate the pre-defined "Heat, excessive" query in BioSense 2.0 using recent Maricopa County, Arizona data; quantify the number of cases retrieved by the query due to chief complaint terms rather than clinical diagnosis; and provide a list of terms to be considered for exclusion criteria while developing a custom query.

\section{Introduction}

Monitoring heat-related illness (HRI) is a public health priority in Maricopa County, Arizona. Since 2006, Maricopa County Department of Public Health has utilized data from hospital discharges, medical examiner preliminary reports, and death certificates to quantify heat-related morbidity and mortality, but these surveillance methods take time. Identifying HRI more quickly would improve situational awareness and allow public health officials to launch a more immediate response to extreme heat events. Arizona began using BioSense 2.0 in July 2014 to collect chief complaint and diagnosis data for syndromic surveillance. The BioSense Front End Application uses a standard query definition for HRI (i.e., "Heat, excessive"), but this definition may perform differently for each jurisdiction.

\section{Methods}

We used BioSense 2.0 to produce a line list of patient records between $1 / 1 / 15$ and $8 / 15 / 15$ that met criteria in the "Heat, excessive" query definition. Records with a clinical diagnosis or injury code (992 or E900, respectively) were considered confirmed HRI cases. We manually reviewed the chief complaint fields of the remaining records and classified each as probable HRI case, HRI ruled out, or undetermined HRI status. We compiled a list of terms that were common among the ruled out cases and determined how the query would perform if we added these terms to the query as exclusion criteria. As a secondary analysis, we determined whether the exclusion criteria would perform differently depending on season.

\section{Results}

The "Heat, excessive" query retrieved 539 Maricopa County, AZ patient records between $1 / 1 / 15$ and $8 / 15 / 15$. Nearly half of the records had a clinical diagnosis for HRI, while 271 records (50.3\%) required a manual review of the chief complaint data. We classified 148 records (27.5\%) as probable HRI cases because they had symptoms consistent with HRI and 32 records (5.9\%) as undetermined because they did not have strong evidence to suggest HRI but could not be ruled out. We ruled out 91 records $(16.9 \%)$ for HRI because their chief complaint was not related to environmental heat exposure. For example, these patients mentioned feeling heat, swelling, redness, and/or pain; using heat and ice for therapeutic reasons; having dental sensitivities to hot and cold; misspelling "heart"; and misspelling "head". We built a list of potential exclusion terms and tested it against the 271 manually reviewed records (Table). The potential exclusion terms were identified in 132 records (27 probable HRI cases; 17 undetermined records; and 88 HRI ruled out). During the cooler months (January - April), these terms were identified in 40 cases, but only one was considered a probable case. During the hotter months (May - August), these terms were identified in 92 records ( 26 probable HRI cases; 17 undetermined records; and 49 HRI ruled out).

\section{Conclusions}

The pre-defined "Heat, excessive" query in BioSense 2.0 allowed us to quantify Maricopa County's HRI burden in a timely manner. The query retrieved 268 records with a clinical diagnosis for HRI and 148 additional cases that had symptoms consistent with HRI in their chief complaint data (i.e., probable HRI). By manually reviewing the chief complaint data, we found that $17 \%$ of the records were not related to environmental heat exposure. The query could be more specific if exclusion criteria were added. Our next steps will be to continue evaluating data through 2015 , determine whether additional terms should be added as inclusion and exclusion criteria, and validate our proposed query definition against both medical records and finalized hospital discharge data. As we refine our query definition for syndromic surveillance, we will increase our capacity to detect and characterize heat-related morbidity in the county.

\begin{tabular}{|c|c|c|c|c|}
\hline \multirow[b]{2}{*}{ Potential Exclusion Terms } & \multirow[b]{2}{*}{$\mathrm{N}(\%)$} & \multicolumn{3}{|c|}{$01 \operatorname{Jan} 15-15$ Aug $15(N=271)$} \\
\hline & & $\begin{array}{c}\text { Probable HRI } \\
\text { case }\end{array}$ & $\begin{array}{l}\text { Undetermined } \\
\text { HRI status }\end{array}$ & HRI ruled out \\
\hline 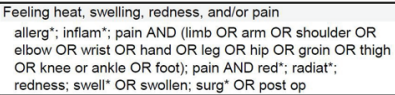 & $87(32.1 \%)$ & 9 & 12 & 66 \\
\hline $\begin{array}{l}\text { Using heat / ice for therapeutic reasons } \\
\text { ibuprofen OR ibuprophen OR alieve OR motrin OR tylenol; } \\
\text { injur" OR trauma; heat AND ice; heat AND (applied OR } \\
\text { tried OR used OR using); (pain AND (back OR neck OR } \\
\text { flank)) OR lumbago; relief OR resolve }\end{array}$ & $67(24.7 \%)$ & 19 & 6 & 42 \\
\hline $\begin{array}{l}\text { Dental pain and sensitivities to hot / cold } \\
\text { dental; hot AND cold; oral AND surgt; pain AND (jaw OR } \\
\text { mouth OR teeth OR tooth); sensitiv" AND (heat OR hot) }\end{array}$ & $10(3.7 \%)$ & 3 & 0 & 7 \\
\hline $\begin{array}{l}\text { Misspelled "heart" } \\
\text { "heat beat"; "heat racing": "heat rate"; palpitations }\end{array}$ & $6(2.2 \%)$ & 0 & 1 & 5 \\
\hline $\begin{array}{l}\text { Misspelled "head" } \\
\text { "hitting heat" }\end{array}$ & $2(0.7 \%)$ & 0 & 0 & 2 \\
\hline $\begin{array}{l}\text { Miscelllaneous } \\
\text { burn AND mouth; "heat flash" OR "hot flash"; heat AND } \\
\text { rash; "heat sensation"; "hot tub"; oven }\end{array}$ & $23(8.5 \%)$ & 1 & 2 & 20 \\
\hline Total cases excluded using all potential terms & $132(48.7 \%)$ & 27 & 17 & 88 \\
\hline
\end{tabular}

Table. Number of Maricopa County, Arizona records that included one or more of the potential exclusion terms

Keywords

BioSense; Evaluation; Heat-related illness; syndrome; query

\section{Acknowledgments}

The authors thank Arizona Department of Health Services for implementing BioSense 2.0 in Arizona.

*Jessica R. White

E-mail: JessicaWhite@mail.maricopa.gov 